\title{
Reducing barriers to care for patients who practise consensual nonmonogamy
}

\author{
Sharon M. Flicker PhD
}

— Cite as: CMAJ 2019 October 15;191:E1118-9. doi: 10.1503/cmaj.191246

See related article at www.cmaj.ca/lookup/doi/10.1503/cmaj.190224

$\mathbf{L}$ inked research by Arseneau and colleagues highlights difficulties faced by individuals in consensually nonmonogamous relationships when they access health care. ${ }^{1}$ Consensual nonmonogamy encompasses a wide variety of relationships that include "the practice of, belief in, or willingness to engage in multiple [intimate], romantic and/or sexual relationships with the informed consent of everyone involved." ${ }^{2}$ Both interest and engagement in consensual nonmonogamy seem to be increasing. ${ }^{3}$ At the same time, training of health care providers to work effectively with this population has not kept up with these trends. Although existing research is limited, several qualitative studies have documented the concerns of patients in consensually nonmonogamous relationships regarding their health care experiences: concerns that may affect their willingness to access health care and concerns about the quality of their care when they do. ${ }^{1,4}$

These individuals face great stigma and are often pathologized based on inaccurate assumptions. ${ }^{5}$ However, several studies have failed to find differences in relationship satisfaction and other indices of relationship functioning between monogamous and consensually nonmonogamous relationships. ${ }^{2,5}$ Furthermore, rates of sexually transmitted infections (STIs) are comparable across consensually nonmonogamous and monogamous populations. ${ }^{6}$ Despite this, the majority of individuals who practise consensual nonmonogamy have experienced discrimination, ${ }^{7}$ a phenomenon associated with higher levels of psychological distress and poorer health outcomes. ${ }^{8}$ Health care providers have an opportunity to mitigate this stress by providing inclusive environments and sensitive health care.

In a recent qualitative study of patients in consensually nonmonogamous relationships, participants reported that their health care experiences were undermined by provider assumptions that patients were monogamous and provider ignorance regarding other relationship structures. ${ }^{4}$ These patients subsequently felt pressure to educate their providers and furthermore experienced unnecessary barriers to obtaining regular, customized STI screenings and individualized advice regarding risk reduction strategies. Several participants who had disclosed that they practised consensual nonmonogamy reported experiencing both implicit and explicit judgment from their provider. Similar

\section{KEY POINTS}

- Mononormativity, or the assumption that clients are and should be monogamous, creates barriers to accessing health care and engaging in effective partnerships with providers for patients who practise consensual nonmonogamy.

- Improved training and continuing education for health care providers is essential for providing optimal health care to this population.

- Using inclusive language on patient history forms, providing space for multiple intimate partners to participate in care, asking open-ended questions, and offering a nonjudgmental stance signal safety to patients in consensually nonmonogamous relationships.

- Including search terms regarding consensual nonmonogamy competency in health care provider directories may increase the likelihood of these patients accessing care and being open with their providers.

concerns were shared by the participants in the linked qualitative study of the birth care experiences of Canadian polyamorous individuals, who reported that the failure to acknowledge and accommodate their multiple intimate partners, both in terms of physical space and on forms, negatively affected their birthing experiences. ${ }^{1}$ Some chose home births as a means of avoiding anticipated hospital restrictions (both physical and administrative) that would have prevented full participation of all their partners in the birth.

Given the limited research among patients who practise consensual nonmonogamy, research with other sexual minorities may further our understanding of health care experiences. Such research suggests that most patients prefer to be out about their identity to their provider in order to receive individualized care, particularly increased STI screening, if relevant, and advice to moderate the risks of their specific behaviours. ${ }^{9}$ However, many reported avoiding or delaying medical care, or failed to disclose potentially medically relevant information to their providers, because of fear of stigma and judgment. This should be of concern to providers, as less than full disclosure of relevant information can lead to poorer health outcomes. Findings from 
studies with other sexual minorities mirror the findings from the studies on patients in consensually nonmonogamous relationships. For instance, in 1 qualitative study of kink-oriented patients, many felt providers had little understanding of nontraditional sexual arrangements, resulting in a failure to assess these relations. ${ }^{9}$ Although many participants who disclosed their identity reported positive responses, others perceived subtle changes in body language, which they interpreted as reflecting negative judgment.

Implicit biases among health care providers have been shown for a wide range of stigmatized identities and are thought to relate to negative health outcomes for members of these groups through 2 mechanisms: this implicit bias affects care-based decisions by providers, and impairs doctor-patient interactions, which subsequently undermines the patient's trust in the provider and engagement and adherence to their treatment. ${ }^{10}$ Providers' implicit bias has been tied to both objective behaviours (rated from audiotapes) and patients' subjective experiences of their provider. ${ }^{10}$ Thus, reducing providers' implicit biases toward sexual minority groups, and patients in consensually nonmonogamous relationships in particular, is vital to addressing health disparities.

Ensuring one's practice is inclusive of individuals in diverse relationship structures starts with the recognition that not all patients are monogamous. Individuals are more likely to reveal concealable stigmatized identities under conditions they deem are safe to do so. Thus, overt messages of inclusivity are important to counteract hesitance on the part of patients to disclose. Explicit indicators of inclusivity include asking about relationship structure during intake, using inclusive language on forms and providing space to list multiple partners, asking open-ended questions, allowing for multiple partners to be present during treatment and promoting risk-informed sexual practices rather than exclusively promoting monogamy. ${ }^{1,4,11}$ Providing sexual health resources as well as accessible and customizable STI screenings with easily shareable results may address unique health needs of patients in consensually nonmonogamous relationships. ${ }^{4}$

To foster effective partnerships with these patients, providers should work to reduce their implicit biases. To this end, medical schools and continuing education programs should address the current lack of training regarding consensual nonmonogamy, which could alleviate the burden that some patients feel to have to educate their provider., ${ }^{1,4}$ In the meantime, to protect themselves from anticipated stigma and judgment, patients in consensually nonmonogamous relationships have expressed the desire to prescreen providers to identify those who are inclusive and affirming of consensual nonmonogamy, and health care organizations can reduce this burden by adding search terms regarding consensual nonmonogamy competency to provider directories. ${ }^{4,11}$ This may serve as a stop-gap measure until training and continuing education programming systemically include both bias-reduction and essential practices to work effectively with patients in consensually nonmonogamous relationships. Finally, although the association between sexual minority status and stigma-mediated health disparities is well documented, ${ }^{12}$ more research is needed to better understand and address this phenomenon among patients in consensually nonmonogamous relationships.

\section{References}

1. Arseneau E, Landry S, Darling EK. The Polyamorous Childbearing and Birth Experiences Study (POLYBABES): a qualitative study of the health care experiences of polyamorous families during pregnancy and birth. CMAJ 2019;191:E1120-7.

2. Rubel AN, Bogaert AF. Consensual nonmonogamy: Psychological well-being and relationship quality correlates. J Sex Res 2015;52:961-82.

3. Moors AC. Has the American public's interest in information related to relationships beyond "the couple" increased over time? J Sex Res 2017;54:677-84.

4. Vaughan MD, Jones $P$, Taylor BA, et al. Healthcare experiences and needs of consensually non-monogamous people: results from a focus group study. J Sex Med 2019;16:42-51.

5. Conley TD, Matsick JL, Moors AC, et al. Investigation of consensually nonmonogamous relationships: theories, methods, and new directions. Perspect Psychol Sci 2017;12:205-32.

6. Lehmiller JJ. A comparison of sexual health history and practices among monogamous and consensually nonmonogamous sexual partners. J Sex Res 2015;12:2022-8.

7. Witherspoon RG. Predictors of perceived stigmatization within consensually nonmonogamous relationships [thesis]. Alhambra (CA): Alliant International University; 2016.

8. Pascoe EA, Smart Richman L. Perceived discrimination and health: a meta-analytic review. Psychol Bull 2009;135:531-54.

9. Waldura JF, Arora I, Randall AM, et al. Fifty shades of stigma: exploring the health care experiences of kink-oriented patients. J Sex Res 2016;13:1918-29.

10. Zestcott CA, Blair IV, Stone J. Examining the presence, consequences, and reduction of implicit bias in health care: a narrative review. Group Process Intergroup Relat 2016;19:528-42.

11. Schechinger H. 5 reasons to include consensual non-monogamy on healthcare provider locator directories. In: Diverse love and relationships [blog]. $2018 \mathrm{Dec}$. 12. Available: www.drheathschechinger.com/blog (accessed 2019 Sept. 21).

12. Institute of Medicine (US) Committee on Lesbian. Gay, Bisexual, and Transgender Health Issues and Research Gaps and Opportunities. The health of lesbian, gay, bisexual, and transgender people: building a foundation for better understanding. Washington (DC): National Academies Press; 2011.

\section{Competing interests: None declared.}

This article was solicited and has not been peer reviewed.

Affiliation: Department of Psychology, California State University, Sacramento, Calif.

Correspondence to: Sharon Flicker, flicker@csus.edu 